\title{
HIGHLIGHTS OF I.A.U. COLLOQUIUM 101: EXCERPTS FROM A PANEL DISCUSSION
}

\author{
Chairman: \\ Sidney van den Bergh \\ Panellists: \\ Craig Wheeler, Rob Fesen, Richard Strom, David Helfand, Carl Heiles
}

Editors' Note: At the conclusion of IAU Colloquium 101 a panel discussion was held. The panellists were asked to give their opinions on significant new developments presented at the Colloquium and to outline questions to be answered in the future. The editors have summarized the discussion.

SIDNEY VAN DEN BERGH: I would like to take a few minutes to give my own impressions of our meeting. A good reference point is the I.A.U. Symposium 101 ("Supernova Remnants and their X-ray Emission") which took place in Venice five years ago. In comparing this meeting with the Venice meeting, it strikes me that the theoreticians now seem to be more realistic; they are no longer talking about a smooth interstellar medium - they have lumps in the medium, and lumps in the supernova remnants. There was a real conundrum in Venice that has now been resolved. At that time there were two kinds of supernovae - Type I and Type II - but there were three kinds of supernova remnants - objects like Tycho, ones like Cas A and objects like the Crab. Now we realize that there are three kinds of supernovae and we seem to agree which kinds of supernovae can produce which kinds of remnants. Another big difference was that five years ago we didn't have SN1987a - this has added a great deal of interest to the present meeting. There are new problems that were brought up at this meeting that were not discussed in Venice. For instance, why is it that many plerions, including the Crab Nebula, don't have a high velocity shell around them?

CRAIG WHEELER: As a person interested in hydrodynamics and the evolution of supernovae I come to a meeting such as this asking "What can we learn from supernova remnants about the kinds of stars that exploded?". At this meeting we have seen some progress on this subject, in particular from the modelling of X-ray spectra (e.g. Smith and Jones, Itoh et al., and Hamilton and Fesen). I would also like to know the energy of a supernova for comparison with the predictions of models - carbon deflagration versus core collapse, and a prompt explosion core collapse versus neutrino pumping. I have learned at this conference that there is no answer yet to this second question. I would also like to know the mass and composition of the ejecta, and I would like to know the frequency of supernovae.

As Dick McCray mentioned, the different types of supernova precursors, depending upon the the amount of mass they shed, where they are, and what they do to the ISM, may, from their winds, make different bubbles and super-bubbles. We are after that global problem - the connection between star formation, winds and bubbles, supernovae and supernova remnants, shocks in the interstellar medium, and once again, star formation. From the point of view of stellar evolution, I think that we are making some progress because we are asking different kinds of questions. I was particularly interested in the questions of supernovae and supernova remnants in or near HII regions (Chu and Kennicutt) and molecular clouds (Dubner and Arnal; Burton; Fürst et al.). 
ROB FESEN: The main theme I've discerned from this conference concerns the importance of the circumstellar material in affecting the local environment and evolution of young supernova remnants, particularly those of Types Ib and II. Lozinskaya showed what a massive star could do to its local interstellar medium - the UV radiation, the stellar winds and wind blown bubbles, and the ejection of slow shells of material. She showed some fine examples of multiple shell structures and one example of a pre-supernova candidate. Rob Braun also showed a perfect example of a wind blown bubble in the cavity next to IC443. Shelled and unshelled Crab-like remnants are, I think, just different phases in the evolution of the same type of object. A "naked Crab" would be one that comes from a massive star progenitor where the shock wave is still moving through the stellar wind cavity. A "shelled Crab" is an older version where the shock wave is now moving through the circumstellar material. I had thought it was going to be impossible to distinguish between remnants of Type I and Type II supernovae, but if Type I's come from white dwarfs, they won't churn up the interstellar medium. This may explain why objects like SN1006 and Tycho form very uniform, smooth shells in the X-ray and radio, and Balmer dominated emission in the optical. Precursors of Type II's really do affect their local ISM and the remnant's early evolution is affected strongly by the mass loss of the progenitor star; Cas A, 3C58 and Kepler are perfect examples (I would call Kepler a Type II rather than $\mathrm{Ib}$ because there is evidence in the X-rays and optical for considerable mass around the remnant).

Long ago, in Peter Shull's observations of the LMC remnants N49 and N63A, he found very high velocity features along filaments near the peripheries of those objects. This may be the same phenomenon that Blair, Chu and Kennicutt are seeing in the high surface brightness remnants in M33 where they have observed extremely high expansion velocities, 200 to $300 \mathrm{~km} / \mathrm{s}$ in what seems to be a fairly large remnant. These might be explained by the theoretical work of Norman et al. which suggests a blast wave interacting with very clumpy circumstellar material. On the other hand the optical images and spectra presented partially here by Hester and Raymond, and the high resolution spectra of Greidanus and Strom for old objects like the Cygnus Loop present a completely different picture; it is the interstellar, not circumstellar medium and it is fairly uniform on the large scale. You can't look at the pictures of Hester and Raymond and think there are a lot of clumps; there really are non-radiative sheets there. I wonder whether we will be able to say anything specific about any one region in the interstellar medium - about its density, about the shock velocity and abundances, or whether we will only be able to discern general trends.

Eli Dwek stressed the importance of dust as a density diagnostic in high temperature supernova remnants. On the other hand, Arendt has looked at IRAS data for a large number of supernova remnants in our galaxy and found only 25 to 30 percent with a fair amount of detectable infrared emission. Is this just a sensitivity problem in removing the complex galactic background or is it really a problem of what sort of medium the large remnants have moved into? Maybe when a supernova remnant encounters a dark cloud you see the infrared emission, but if it does not, like SN1006, then you simply don't see the infrared emission. Three more things. I was really impressed with (i) the large number of supernova remnants discovered optically by Long et al. in a small region of M33, (ii) the work on Puppis A of Winkler et al. showing that the ejection blobs of remnants last at least 3600 years, which is very surprising, and (iii) the work of Ballet et al., Jerius and Teske, and Brown et al. on the coronal line emission which they try to match spatially with the radiative or X-ray gas. 
I have a list of questions for the future which the people in the optical, UV, and infrared will have to answer. (i) Where is the Crab's outer shock? Why can't we confirm or deny its existence? (ii) If Cas A really was a Wolf-Rayet star, where is its wind blown bubble? The star should have produced a big bubble, and we don't see much evidence for one. (iii) Where are all the other $O$ stars around these young remnants? (iv) Cas $A$ simply cannot be the youngest remnant in our galaxy - it is 300 years old - where are all the other ones? (v) Where is the blast wave in Cas A - is it ahead of the knots or have the knots punched through? (vi) Finally there is the problem of CTB80. It is not a Crab and it is not a shell, it is not a regular remnant, but it certainly is a supernova remnant and it has an X-ray point source, but it is not clear how it fits into the whole picture.

TATJANA LOZINSKAYA: I would like to say something about the fast shell around the Crab nebula. We can speculate that we are beginning to see a shell-like radio source around the Crab nebula because there are some hints that the spectrum is steeper and the polarization smaller on the periphery. If so, then the remnant is not "Crab-like", but a composite shell-Crab showing the very beginning of a radio synchrotron shell. If I am right (I don't insist) then the question arises - where does the turbulent amplification of the magnetic field take place? If it is between ejected and swept-up gas then the Crab is peculiar because of the small expansion but if it is between the pulsar driven plasma and the ejecta then we might expect to see the fast shell.

RICHARD STROM: I thought I would begin by saying just what radio emission tells us. The one great advantage of these long wavelengths is the lack of extinction or, as radio astronomers might say, absorption. The second thing about radio emission is that it is a good tracer of shock fronts and indicates the presence of magnetic fields. The final thing is that radio photons, being low energy quanta, are plentiful and that is why radio surveys, such as the one described by the Bonn group (Reich et al.) still provide the best way of finding new galactic supernova remnants. You might ask, should we be looking for more? My answer is yes. Further searches should turn up more bizarre and rare types and it may help us to correct biases in catalogs. They may also reveal some young supernova remnants, ones that are just turning on. If one supernova explodes in the galaxy every 50 years or so, there must be some out there which are now brightening. In Roger Chevalier's review we heard about the models of radio emission from young remnants - it seems to me that the radio light curves will be useful as probes of circumstellar conditions.

There has been some very beautiful work describing radio line emission, primarily HI and millimetre molecular lines. I think we are just about to see a great expansion in the millimetre work, with several telescopes that have just come on line. We have seen some lovely examples of emission from IC443 - one wonders why people haven't been looking there to discover new species because it seems to be so rich in molecules.

At Symposium 101 five years ago we felt that the radio $\Sigma-D$ relationship, certainly as a distance indicator, was finished, but I see it has tentatively come back this time, in Colloquium 101, in Elly Berkhuijsen's talk, and in Jim Caswell's.

I've read many papers in which the first statement is that magnetic fields are dynamically unimportant so we can ignore them. In Don Cox's talk, we heard of the importance of the magnetic pressure in the interstellar medium. I was glad to hear in Roger Blandford's talk about the modelling of shocks in which magnetic fields, quasi-parallel and quasi-perpendicular, are included. Radio observations can help to locate the shock fronts - we should also attempt to understand more about the role of the magnetic fields. Some questions. Should we carry out more surveys or should we try to understand the 
remnants that we already have? Are all extended non-thermal galactic radio sources supernova remnants? And when do supernova remnants, as distinct from the supernovae themselves turn on as radio sources?

DAVID HELFLAND: I can answer one of the questions. We know that all non-thermal radio sources in the galaxy are not supernova remnants.

CARL HEILES: Do we know that all supernovae form remnants that are observable in the radio?

\section{RICHARD STROM: We don't know.}

CRAIG WHEELER: There were several poster papers that tried in various ways to estimate how many supernova remnants of the kind that are observed actually exist in the galaxy - the numbers are between 700 and 1200 . It seems to me we should keep hunting for these things.

DAVID HELFAND: I would like to thank the previous speakers for not leaving me much time because there isn't much to say. We haven't learned anything new about the X-ray emission of supernova remnants in some time - or at least we don't have any new data to talk about. Only twelve out of the seventy contributed papers were in any way related to X-rays, which is simply a statement that there is a need for the capability for making more X-ray observations, and I think that further observations are going to be useful. Cox and McKee and McCray and Dopita convinced everyone that to understand how supernovae interact with the interstellar medium we probably have to go to larger scales than supernova remnants themselves. We probably will find many more old ones and they are likely to tell us more again about the interstellar medium. Going to larger scales can include X-ray emission; there was a fascinating poster on the X-ray emission of the Large Magellanic Cloud (Helfand, Wu and Wang, not published) which might lead us to test some of the global models of the interstellar medium which we heard a significant amount about. On the other hand, if we are talking about Craig Wheeler's question "what can the supernova remnant emission tell us about the supernova?" then our hopes must be pegged on a more detailed study of the half dozen very young remnants that we know already.

A comment to what Rob Fesen said about the naked Crabs and clothed Crabs. It should be noted that the second most X-ray luminous Crab-like object out of the seventeen or eighteen that are known (G29.7-0.3) is inside a shell and without a doubt the X-ray emission tracks the current energy output of the neutron star since the particle lifetimes are only a year or so. That would suggest it is probably not just an age effect that distinguishes between the two - it probably has more to do with the progenitor and perhaps the surrounding medium.

Now, to come to the future and what needs to be done in X-ray astronomy. There are two or three planned missions as most of you know, which I would say don't match very well the crucial questions which we need to answer for the study of supernova remnants. I think what we need for this subject is spatially resolved moderate and high resolution $\mathrm{X}$-ray spectroscopy and unfortunately none of the missions planned before the end of the century really delivers that. We don't need 1 arcsecond resolution for spectroscopy, we need 1 arcminute resolution and the reason is very simple - there just aren't enough photons for any reasonable size detector to use 1 arcsecond resolution. That is the only way we are really going to determine the key things about supernova remnants which $\mathrm{X}$-rays can tell us: the temperatures, the densities, and the compositions of the gas and maybe even the kinematics given high enough resolution. Therefore I cannot see an optimistic outlook for the future of X-ray observations of supernova remnants. 
CARL HEILES: Q stands for questions, and I have four of them here; $Q$ also stands for the fraction of the volume of the interstellar medium that is filled by the hot ionized medium of McKee, Ostriker, Cox and Smith. The four questions relate to Q. Question 1: What fractions of supernovae are of Type Ia and of Type Ib? This is important because SNe Type I were thought to be randomly distributed in space and time and would have produced individual bubbles that were uncorrelated. The problem in the past has been that the derived rates of Type I combined with the energies which we believed they had, meant that the $Q$ value due to these remnants alone was so large that many observers didn't believe it. We now have two sub-types of Type I's and only the Ia's will produce these uncorrelated bubbles. Since the frequency of Type Ia's is smaller, so is the value of $\mathrm{Q}$. The $\mathrm{Q}$ for these uncorrelated supernovae also depends on the explosion energy and we have heard several values for that energy here this week.

Question 2 has to do with the other type of supernova - the type that are correlated in space and time. They are correlated in two ways. First, they are correlated with each other and second, they are correlated with molecular clouds - something that has been made very clear at this meeting, in a more general sense by the paper of Lozinskaya, and also in a number of individual papers of which the most spectacular one was by Fukui and Tatematsu. When one of these clusters of supernovae goes off, instead of blowing a spherical bubble in the interstellar medium, there is enough energy to punch through the plane and you have to consider the fraction of the area of the plane filled with hot bubbles. We have seen calculations by Tomisaka and Ikeuchi, and by MacLow et al. of this effect, which occurs only if the energy released by the combined effects of a cluster is enough to actually break through the plane. That depends on (a) the energy per supernova, and (b) the number of stars per cluster that will go supernova. In order to avoid an embarrassingly large $Q$ value for these correlated supernovae we can put many more stars per cluster so that we reduce the number of clusters that go off and thereby reduce the number of holes in the plane.

Another way to accomplish this might be through the association with molecular clouds that we have heard about. This leads me to my next question. Question 3: I would like to know if a typical molecular cloud in the galaxy or a typical Giant Molecular Cloud is large enough to act as a significant condensation centre to soak up an appreciable fraction of the energy which would otherwise go into producing these holes.

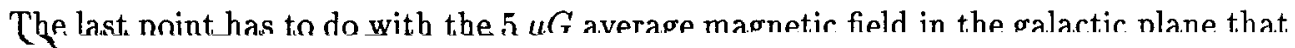


CARL HEILES: That is an extremely good point and I think your work is very important. The fact that supernovae of these types are correlated with molecular clouds means that some fraction will go off inside molecular clouds and those molecular clouds will essentially soak up all the energy. Here I was referring to the case where you have supernovae sitting outside the molecular cloud - that is a problem related to the one you treated. I would like to see a good treatment of this.

\section{REFERENCES TO PAPERS IN THIS VOLUME:}

Arendt, R. G., page 379.

Ballet, J., Caplan, J., Rothenflug, R., and Soutoul, A., page 411.

Berkhuijsen, E. M., page 285.

Blair W. P., Chu Y.-H., and Kennicutt, R. C., page 193.

Blandford, R. D., page 309.

Braun, R., page 227.

Brown, L., Woodgate, B. E., and Petre, R., page 407.

Burton, M. G., page 399.

Caswell, J. L., page 269.

Chevalier, R. A., page 31.

Chu, Y.-H., and Kennicutt, R. C., page 201.

Cox, D. P.,page 73.

Dopita, M. A., page 493.

Dubner, G. M., and Arnal, E. M., page 249.

Dwek, E., page 363 .

Fukui, Y., and Tatematsu, K., page 261.

Fürst, E., Reich, W., Hummel, E., and Sofue, Y., page 253.

Greidanus, H., and Strom, R. G., page 443.

Hamilton, A. J. S., and Fesen, R. A., page 59.

Hester, J. J., and Raymond, J. C., page 415.

Itoh, H., Masai, K., and Nomoto, K., page 149.

Jerius, D., and Teske, R. G., page 145.

Long, K. S., Blair, W. P., Kirshner, R. P., and Winkler, P. F., page 197.

Lozinskaya, T. A., page 95.

MacLow, M.-M., Norman, M. L., and McCray, R. A., page 461.

McCray, R., page 447.

McKee, C. F., page 205.

Norman, M. L., Dickel, J. R., Livio, M., and Chu Y.-H., page 223.

Reich, W., Fürst, E., Reich, P., and Junkes, N., page 293.

Smith, B. W., and Jones, E. M., page 133.

Tomisaka, K., and Ikeuchi, S., page 477.

Winkler, P. F., Tuttle, J. H., Kirshner, R. P., and Irwin, M. J., page 65. 